\title{
Estructura demográfica y desempeño financiero municipal en el departamento de Boyacá, Colombia
}

\author{
Demographic Structure and Municipal \\ Financial Performance in Boyacá, Colombia \\ Estrutura Demográfica e Desempenho \\ Financeiro Municipal em Boyacá, Colômbia
}

Luis Felipe Fajardo Pineda*

Fecha de recepción: 4 de marzo de 2019

Fecha de aceptación: 4 de julio de 2019

Cómo citar este artículo/ To reference this article / Comment citer cet article / Para citar este artigo:

Fajardo-Pineda, L. (2019). Estructura demográfica y desempeño financiero municipal en el departamento de Boyacá, Colombia. Apuntes del CENES, 38(68), 247 - 272. https://doi.org/10.19053/01203053. v38.n68.2019.9014

* Economista y administrador público con especialización en Finanzas Públicas. Asesor y capacitador de entidades territoriales en el departamento de Boyacá e investigador junior de la Escuela Superior de Administración Pública - ESAP. Tunja, Colombia. luisfelipe.fajardopineda@gmail.com. Q http://orcid.org/0000-0001-6250-7980 


\section{Resumen}

En este escrito se analiza la correlación entre la estructura demográfica de los municipios del departamento de Boyacá, Colombia, y su desenvolvimiento financiero para el año 2015. Se busca determinar teórica y empíricamente si existe relación de correspondencia entre la reducida población observada en las entidades municipales del departamento y el desempeño financiero de las alcaldías, mediante un estudio cuantitativo de correlación lineal entre las dos variables citadas, a partir de datos institucionales y apoyando los resultados con los postulados teóricos de relevancia. Se concluye que hay una fuerte relación entre el tamaño poblacional y el desenvolvimiento fiscal de las entidades del departamento, donde la primera variable genera una tendencia negativa en los indicadores financieros municipales, lo que implica grandes retos institucionales para las entidades.

Palabras clave: disminución de la población, descentralización, hacienda local, administración local, presupuesto y gasto de la administración estatal, autonómica y local, administración pública.

Clasificación JEL: H70, H71, H72, H61, H83. 


\begin{abstract}
In this paper we analyze the correlation between the demographic structure of the municipalities of the state of Boyacá (Colombia) and their financial development for 2015. The aim is to determine theoretically and empirically if there is a correspondence between the reduced population observed in the municipal entities of Boyacá and the financial performance of the municipalities, by means of a quantitative study of linear correlation among the two variables cited, from institutional data, supporting the results with the relevant theoretical postulates. We conclude that there is a strong relationship concerning the population size and the fiscal development of the entities of the department, where the first variable generates a negative trend in municipal financial indicators, which implies immense institutional challenges for the entities.
\end{abstract}

Keywords: population decline, decentralization, local finance, local government, State and Local Budget and Expenditures, Public Administration. 


\section{Resumo}

O artigo analisa a correlação entre a estrutura demográfica dos municípios do departamento de Boyacá-Colômbia e seu desenvolvimento financeiro para o ano de 2015. O objetivo é determinar teoricamente e empiricamente se há uma correspondência entre a população reduzida observada nas entidades municipais do departamento e do desempenho financeiro dos municípios. Para este fim, um estudo quantitativo da correlação linear entre as duas variáveis citadas é implementado a partir de dados institucionais, apoiando os resultados com os postulados teóricos relevantes. Assim, o artigo conclui que existe uma forte relação entre o tamanho da população e o desenvolvimento fiscal das entidades do departamento, onde a primeira variável puxa negativamente os indicadores financeiros municipais, gerando grandes desafios institucionais para as entidades.

Palavras chave: redução da população, descentralização, tesouraria local, administração local. 


\section{INTRODUCCIÓN}

Uno de los resultados de las reformas sociopolíticas que vivió el Estado colombiano a finales del siglo $\mathrm{XX}$ es la institucionalización de la descentralización como el modelo de organización y distribución de competencias que garantizaría una mayor proximidad entre gobernantes y gobernados. Pieza fundamental del proceso sería la consolidación del municipio como célula básica de la arquitectura estatal, con atribuciones políticas, administrativas y particularmente financieras, como la participación en los ingresos corrientes de la nación, autonomía en el ejercicio presupuestal y disponibilidad para aprobar un sistema tributario propio (Pinilla, Jiménez \& Montero, 2015). Así, la consigna ideológica del proceso sería la ratificación de los gobiernos locales como niveles institucionales de generación de desarrollo en cada uno de los territorios del país, mediante el desenvolvimiento fiscal $\mathrm{y}$ administrativo de los mismos.

No obstante, tras cerca de tres décadas de inicio constitucional del proceso, la gran mayoría de entidades territoriales en Colombia no ha desarrollado significativamente sus finanzas, pues existe gran dependencia de ingresos como las transferencias para solventar su financiamiento. En efecto, en la implementación de la descentralización se trasladaron competencias sobre gran parte de los gastos sociales del Estado hacia las administraciones locales, pero se concedieron reducidas bases tributarias para su financiamiento, lo cual generó un uso excesivo del crédito territorial y, por ende, una crisis en las finanzas públicas del país (Pening, 2003). Como resultado de ello, las entidades territoriales asumen al comienzo del presente siglo los compromisos fiscales consagrados en las reformas de saneamiento fiscal, que se resumen en la racionalización del gasto, autofinanciamiento de los egresos para funcionamiento, reducción de pasivos, entre otras (Sánchez, Naranjo \& Rincón, 2005). Se consolida así un modelo de recentralización fiscal, el cual minimiza la flexibilidad financiera de las entidades públicas en el orden local. 
Es necesario señalar que este contexto problemático tendría un desenvolvimiento heterogéneo entre los distintos territorios del país, que mostraría una clara inequidad en las estructuras de ingresos y gastos de las distintas entidades locales. Los resultados interanuales de desempeño territorial presentados por el Departamento Nacional de Planeación (DNP) indican que las finanzas municipales se desenvuelven de forma diferencial, situando variables como la categoría, los niveles de desarrollo o la ubicación geográfica de los municipios como elementos de clasificación y diferenciación. De hecho, tal como lo demuestra la evidencia empírica en los países latinoamericanos, las falencias estructurales de los procesos de descentralización fiscal se agudizan en ambientes locales particulares, donde el recaudo de ingresos se hace mucho más complejo ante el reducido dinamismo económico y demográfico (CEPAL, 2016). Entre las posibles causas de esta divergencia se pueden resaltar la estructura económica, la complejidad cultural, la ubicación geográfica, el desempeño institucional o el tamaño poblacional.

Para este caso, el documento enfatiza su análisis en la conducta demográfica, por lo que el objetivo del mismo es identificar teórica y empíricamente la relación de las finanzas públicas en el nivel municipal en el departamento de Boyacá frente a la estructura demográfica de sus entidades. El uso de esta zona para el estudio obedece a la aguda tendencia de despoblación que han padecido di- versos municipios boyacenses (Pineda, 2016), lo cual la convierte en un caso propicio y particular para identificar su relación en el escenario financiero. De igual manera, se aplica el análisis para el año 2015, porque este corresponde a la última vigencia del periodo de gobernantes municipales, tiempo en el cual se materializan los principales procesos de planeación pública, por lo que este año permite evidenciar claramente la capacidad financiera de las entidades.

Para alcanzar el objetivo planteado, se desarrolla un análisis cuantitativo de correlación lineal entre dos variables: tamaño poblacional y desempeño fiscal municipal, mediante el empleo de los datos poblacionales emitidos por los diferentes procesos censales ejecutados por el Departamento Nacional de Estadística (DANE), así como de los resultados de desempeño fiscal e integral presentados por el DNP sobre los 123 municipios de Boyacá en el periodo de análisis. A partir de ello, se observa si la estructura demográfica de las entidades boyacenses, caracterizada por el reducido tamaño poblacional (mayoritariamente de menos de 10000 habitantes), altera el desenvolvimiento financiero de las administraciones territoriales correspondientes.

De esta manera, se plantea como primera medida un diagnóstico general de la descentralización fiscal colombiana, desde una perspectiva teórica y empírica; luego se caracteriza la estructura demográfica del departamento de Boya- 
cá, mediante la evaluación de los datos censales del DANE; posteriormente, y haciendo uso de las cifras financieras y de desempeño otorgadas por el DNP, se expresa gráfica y textualmente la conducta fiscal de los municipios del departamento, categorizando la misma por su tamaño poblacional para dimensionar posibles divergencias; por último se presenta una reflexión sobre los factores que inciden en la preeminencia del fenómeno problemático, se proponen alternativas de mejora desde las administraciones públicas y se exponen las conclusiones respectivas.

\section{DESCENTRALIZACIÓN FISCAL Y FINANZAS PÚBLICAS LOCA- LES EN COLOMBIA}

La descentralización fiscal ha sido un proceso de reforma estatal emprendido por la gran mayoría de Estados latinoamericanos a finales del siglo XX, la cual se caracteriza fundamentalmente por la ampliación de las atribuciones estatales de los establecimientos públicos del nivel local. Autores como Asela (2015) la sintetizan como la transferencia de competencias intitucionales a entidades de orden territorial, la cual debe ir acompañada de cierta autonomía política, administrativa y fiscal. En general, la descentralización se puede considerar como un medio para profundizar la actividad estatal a lo largo y ancho del territorio.

No obstante, algunas corrientes del pensamiento heterodoxo han sustentado cómo la estrategia dogmática del nuevo liberalismo económico ha cooptado la descentralización como una herramienta para fortalecer los preceptos del libre mercado. Para autores como Giraldo (2009), la descentralización favorece la libre competencia en la medida que viabiliza la reducción de la intervención estatal sobre las dinámicas económicas, sometiendo su actividad pública a la destinación directa de recursos hacia la población sin capacidad acceder al mercado.

Como muestra de ello, distintos organismos multilatelares como el Banco Mundial (BM), la Organización Mundial del Comercio (OMC) o la misma Comisión Económica para América Latina y el Caribe (CEPAL), han generado tendencias geopolíticas mundiales para aplicar el modelo de subsidio a la demanda en los distintos países emergentes. Modelo que es definido como la política de gasto social que destina recursos estatales directamente a la población vulnerable, de tal manera que se le otorgue al ciudadano la facultad de decidir sobre los bienes sociales que ha de demandar, vinculándose al mercado como un actor potencial. Bajo tales condiciones, la descentralización funge como el mecanismo propicio para insertar este modelo en los distintos regímenes políticos, dado que va a garantizar la distribución territorial del subsidio a la demanda.

Bajo esta premisa se consolida la estrategia de aumentar la proximidad entre el Estado y el demandante de bienes 
púbicos, en la que el municipio se inserta como un agente de vital importancia para el éxito del proceso. Si bien la lógica de la descentralización fiscal es garantizar la focalización del gasto público, se hacía necesario entonces fortalecer las intituciones del orden territorial como establecimientos estatales más cercanos a la población (tal como lo son las alcaldías o las gobernaciones), para garantizar así la distribución eficiente del erario público en el nivel local. En concordancia, Velasco (2007) sostiene que la lógica de la descentralización fiscal se resume en un gobierno central que transfiere recursos y una entidad local administradora, muy allegada a los individuos racionales, quienes eligen la mejor opción de compra de bienes antes suministrados directamente por el Estado.

Las implicaciones de este contexto paradigmático para el tema del presente artículo son principalmente dos: el modelo paternalista de descentralización y el desincentivo para el desenvolvimiento financiero de las entidades locales. Para el primer caso, en la descentalización, el sector central de Gobierno conserva las directrices de política y de gasto público, asignando recursos a las entidades territoriales vía transferencias, las cuales se caracterizan por su condicionalidad; además, dispone para las localidades los tributos con el sistema de recaudo más complejo, por lo que los recursos propios de estas no se dinamizan. Así, a pesar de existir una distribución por competencias entre niveles de gobierno y cierto reconocimiento de autonomía financiera, la inflexibilidad en el presupuesto territorial obliga a que las entidades locales generen gasto público en destinaciones previamente establecidas por el Gobierno nacional, sustentando el paternalismo. Ahora, en lo relacionado con la segunda implicación del modelo, los gobiernos centrales tienden a favorecer criterios como la pobreza o el tamaño poblacional para asignar las participaciones o transferencias; consecuencia de ello es que las entidades no tengan el incentivo para asumir el costo político de incrementar el recaudo en sectores como la propiedad (impuesto predial unificado) o la actividad económica (impuesto de industria y comercio). En síntesis, estos fenómenos demuestran que el modelo privilegia la descentralización como medio propicio para asignar el subsidio a la demanda, sin favorecer con ello el desarrollo financiero de las entidades territoriales bajo el principio de autonomía.

En este contexto, la nación colombiana emprendió la descentralización fiscal hacia finales de los años ochenta del siglo XX, estableciendo distintas atribuciones financieras a las entidades territoriales. Inicialmente, el proceso se formuló como una herramienta para contrarrestar la crisis de la deuda padecida en esta década y transferir la responsabilidad del gasto social a las entidades territoriales. Así, las tres escalas de gobierno estarían definidas, según sus funciones, en defensa, relaciones internacionales, transporte, producción 
y desarrollo para el nivel central de gobierno; industria, agricultura, salud y educación para niveles intermedios del Estado; y, por último, la prestación de servicios públicos se llevaría a cabo por las administraciones municipales (Muñoz, 2013).

A pesar de ello, la implementación de la descentralización fiscal ha estado minada por una serie de restricciones estructurales que limitan el margen de flexibilidad financiera de las entidades municipales. En concordancia, el Estado ha asignado a las entidades territoriales los tributos con menor base impositiva, por lo cual son aquellas de menores niveles de recaudo (Giraldo, 2009). También lo expresan Delgado y Acero (2015), al demostrar que las transferencias intergubenamentales dirigidas a entidades descentralizadas se caracterizan por su naturaleza condicionada y que los impuestos propios no son suficientes para garantizar la actividad pública de los municipios. De hecho, en documentos del Banco Mundial (Boadway \& Shah, 2009) se manifiesta la pertinencia de las transferencias intergubernamentales condicionadas en una estructura descentralizada, entendiendo que la restricción en la destinación del gasto ofrece mayor eficiencia en la ejecución de los recursos estatales.
Ahora bien, las cifras fiscales en Colombia ratifican la carencia de estándares de eficiencia y autonomía financiera en las entidades territoriales, sobre todo en aquellas de menor categorización municipal. Bonet y Ayala (2015) sintetizan que el $70 \%$ de los ingresos tributarios del Estado corresponden al Gobierno nacional central, $20 \%$ a los distritos y un $10 \%$ a todos los 32 departamentos y 114 municipios restantes del país. De igual forma, frente a la evaluación de los resultados de dependencia de las transferencias en los indicadores de desempeño fiscal del 2015, se manifiesta que el indicador ponderado por grupos más alto se presentó en los municipios de sexta categoría con un $68 \%$ promedio de dependencia ${ }^{1}$ (Departamento Nacional de planeación [DNP], 2016b). En otras palabras, la reducida autonomía fiscal se agudiza en los territorios de menor dinámica financiera, demográfica, económica y geográfica, teniendo en cuentas que estas son las cuatro variables que definen la categorización municipal colombiana, según lo establecido en la Ley 1551 de 2012.

Ante estas condiciones, Wiesner (2002) define el concepto de "endogeneidad del gasto territorial" como la principal problemática de la descentralización fiscal colombiana, entendida esta como la excesiva dependencia del gasto de las transferencias del orden nacional

1 Es necesario tener en cuenta que el indicador referenciado del DNP calcula solamente el peso porcentual de los recursos del Sistema General de Participaciones (SGP) y del Sistema General de Regalías (SGR) sobre los ingresos totales, por lo cual no se contabilizan otros tipos de ingresos exógenos como las transferencias departamentales, recursos del FONPET, créditos o aquellos provenientes de procesos de cofinanciación. Ante ello, es de esperar que el indicador de dependencia de recursos externos en los municipios de sexta categoría sea mucho más alta. 
en administraciones locales. Se demuestra que las reformas estatales de este proceso no han consolidado en las localidades márgenes de desenvolvimiento financiero propio, ante lo cual se mantiene centralizada la destinación del gasto. Así, siguiendo los principios del paradigma del mercado, la descentralización en Colombia ha desarticulado la realidad territorial de las reformas a la arquitectura estatal, errando en los mecanismos usados para maximizar las aptitudes financieras de las entidades subnacionales, sobre todo en aquellas de menor dinamismo socioeconómico. Sobre dicha condición se profundiza a continuación con el caso boyacense.

\section{DESPOBLACIÓN Y TAMAÑO POBLACIONAL MUNICIPAL EN EL DEPARTAMENTO DE BOYACÁ}

Desde una perspectiva teórica, el contexto financiero de una entidad pública está directamente ligado a las condiciones demográficas del territorio administrado. El desempeño fiscal depende fundamentalmente de la generación de recursos propios, los cuales se representan en tributos; para el recaudo de los mismos, más allá del esfuerzo administrativo para ejercer el cobro, el tamaño poblacional de la entidad territorial es un elemento clave para establecer el nivel de ingresos propios de las entidades. En este caso, tal como se sustenta en vertientes del pensamiento social como la nueva geografía económica, la aglomeración de factores productivos en un territorio específico conduce hacia la gestación de externalidades positivas, maximizando distintos entornos económicos como el caso de las finanzas públicas (De Lucio, 1997). Así, en caso de presentarse aglomeración del factor trabajo en un municipio, lo cual se puede reconocer como afluencia de población en edad productiva, se produce un desenvolvimiento progresivo de distintos escenarios sociales como el comercial, urbano, de servicios, industrial, entre otros; todos ellos llevan de por medio la responsabilidad tributaria y, por ende, el beneficio hacendístico de las entidades estatales de la jurisdicción.

Teniendo en cuenta esta premisa teórica, Galvis y Meisel (2011) afirman que Colombia padece una pobreza espacial progresiva en algunos de sus territorios, dado que el aislamiento geográfico observado conduce a formar las denominadas "trampas de pobreza", es decir, regiones rezagadas sin aglomeración productiva $y$, por ende, sin generación de externalidades positivas en este sentido. Entre las diveras características diferenciales de estos territorios, el reducido tamaño poblacional sobresale ante evidentes procesos de emigración. De esta manera, se puede considerar que el desarrollo de procesos como la descentralización fiscal toma distintos matices en ambientes demográficos particulares.

Ahora, para el caso de Boyacá, la evidencia empírica demuestra el padecimiento de un proceso histórico de des- 
población en regiones específicas, con indudables fenómenos de emigración externa e interna. Según datos del (DANE, 2005b), Boyacá es el departamento con mayor proporción de personas nativas que han emigrado a otras regiones del país, de tal manera que el $34.33 \%$ de los nacidos en territorio boyacense se desplazaron a otra zona del país. Ante ello, Pineda (2016) expresa que la participación promedio de Boyacá en la población nacional ha caído de $8.47 \%$ en 1938 a apenas $3.07 \%$ en el 2005. Así mismo, el autor evidencia una divergencia interprovincial en el comportamiento demográfico del departamento desde mediados del siglo $\mathrm{XX}$, donde las provincias centrales han aumentado su población, mientras que en la periféricas se ha reducido notablemente. Así pues, el autor demuestra que provincias como Centro, Sugamuxi o Tundama han aumentado su población desde 1964 a 2015 en proporciones de $104 \%, 42 \%$ y $63 \%$ respectivamente, mientras que en otras regiones como Norte o Gutiérrez la misma se disminuyó en $47 \%$ para ambos casos en el mismo periodo.

Como consecuencia de ello, la estructura demográfica boyacense tiene una particularidad latente que se puede denominar como sobremunicipalización del territorio, en relación con la reducida cantidad de habitantes por cada uno de sus municipios. En efecto, según datos proyectados del DANE (2005a), en 2016 Boyacá es el segundo departamento del país con el mayor número de municipios (con 123), superado solamente por Antioquia que tiene $125^{2}$. Así mismo, es el cuarto departamento con menor número de habitantes por municipio con 10391 personas promedio, solo más alta que Guainía, Amazonas y Vaupés, territorios claramente particulares por su contexto socioeconómico y geográfico. Este diagnóstico se hace mucho más evidente en la Figura 1, en la cual se demuestra que Boyacá ostenta una mayor proporción de municipios con reducida población (colores rojo y amarillo), que aquella proporción evidenciada en el resto del país. En general, para el 2015 Boyacá cuenta con 62 municipios de menos de 5000 habitantes, 38 entre 5000 y 10000 personas, 15 entre 10000 y 20 000 , y solo ocho municipalidades que superan los 20000 residentes. En esta medida, el departamento se caracteriza por contener municipios poblacionalmente reducidos.

$\overline{2}$ Aunque Antioquia es un departamento con un tamaño poblacional y una extensión total que triplican el boyacense. 
BOYACÁ

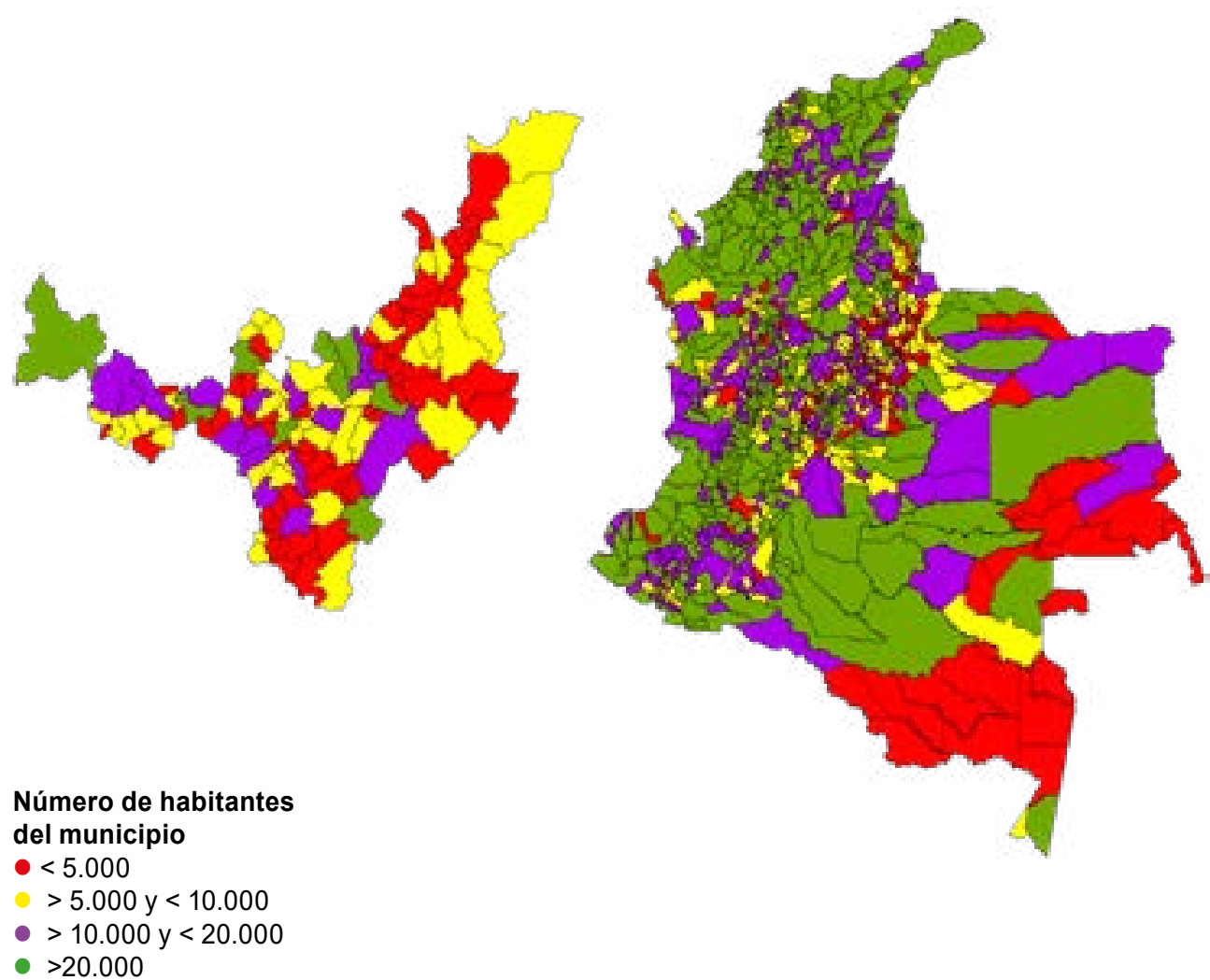

Figura 1. Comparativo tamaño poblacional por municipio. Colombia y Boyacá 2015.

Fuente: elaboración propia con base en datos DANE (2005a).

Así, las conductas sociodemográficas del departamento demuestran un proceso de despoblación en diversas provincias, el cual ha dejado como saldo el reducido tamaño poblacional de gran parte de los municipios del territorio. Si a ello se suma el hecho de que la gran mayoría de las entidades territoriales bajo esta condición ostentan unas estructuras demográficas inminentemente rurales y dispersas, las consecuencias administrativas y financieras para las entidades públicas se complejizan sustancialmente en la región boyacense. Ante ello, se hace necesario evaluar los indicadores de desempeño financiero como insumo para determinar la relevancia de estos procesos demográficos en las finanzas públicas municipales del departamento. 
EVALUACIÓN DE DESEMPEÑO FINANCIERO MUNICIPAL SEGÚN TAMAÑO POBLACIONAL EN BOYACÁ

Dadas las condiciones anteriores, a continuación se analizan los resultados de desempeño fiscal e integral emitidos por el DNP sobre el año 2015, ambos contrastados con el tamaño demográfico de los municipios del departamento. Pero, antes es necesario hacer una aclaración conceptual sobre los indicadores que se van a considerar en el análisis, especificando su objetivo, componentes, categorías y forma de medición. De igual manera, previamente se deben presentar algunas consideraciones sobre el periodo elegido para el estudio.

\section{Desempeño fiscal y desempeño inte- gral: especificación conceptual}

El desempeño fiscal es una valoración en escala de 1 a 100 emitida anualmente por el DNP, en la cual se sintetizan los indicadores financieros de relevancia para categorizar la actividad fiscal de las entidades territoriales. El mismo se configura como el principal medio de comparación y análisis de desempeño financiero municipal en Colombia. El indicador general se subdivide en seis variables específicas, las cuales condensan las aptitudes financieras primordiales que debe desarrollar una entidad pública: autofinanciamiento de gastos de funcionamiento, magnitud de la deuda, porcentaje de ingresos que corresponden a las transferencias, por- centaje de ingresos que corresponden a recursos propios, porcentaje del gasto total destinado a inversión y capacidad de ahorro (DNP, 2015a).

Para clasificar los resultados cuantitativos del desempeño fiscal, el DNP ubica cinco categorías que permiten identificar el estado de las finanzas de cada entidad analizada. Las entidades con el mejor nivel de desempeño, es decir, aquellas que obtengan un puntaje superior a 80 , son catalogadas como solventes, identificadas como organizaciones con las aptitudes idóneas de gastos, ahorro, inversión y endeudamiento. Como un segundo grupo, las entidades con puntajes entre 70 y 80 , se catalogan como sostenibles, caracterizadas con las mismas condiciones del grupo anterior, pero con resultados más bajos. En tercer lugar, las entidades identificadas como vulnerables, con puntajes entre 60 y 70 , son caracterizadas por el DNP como aquellas que cumplen con los requisitos legales de gasto, pero que padecen dependencia fiscal ante la reducida generación de recursos propios. En cuanto al rango de desempeño fiscal entre los 40 y los 60 puntos, se identifican como municipios en riesgo aquellas entidades altamente dependientes con probabilidad de incumplir con los límites de la Ley 617 del 2000. Por último, se catalogan como alcaldías en deterioro financiero aquellas que obtengan puntajes menores a 40 , con alta dependencia, imposibilidad para solventar sus gastos de funcionamiento y problemas para 
desarrollar procesos de inversión (DNP, 2015a).

Por su parte, el desempeño integral combina una serie de variables que manifiestan globalmente el desenvolvimiento de las entidades desde los distintos sectores de gestión. Presenta un balance general de la conducta administrativa de las entidades. El desempeño integral es un indicador propicio para complementar los resultados del desempeño fiscal, ofreciendo una visión más global de las entidades. Este indicador y el desempeño fiscal componen los principales insumos para valorar el desenvolvimiento financiero e institucional de las entidades públicas locales del país, hecho por el cual se han usado para el presente análisis (DNP, 2015b).

También hay que resaltar que la importancia de usar el 2015 como año de análisis obedece a circunstancias políticas y de planeación. En finanzas públicas, el ciclo político es un elemento que altera de forma significativa la actividad estatal. Este se define como una tendencia propia de los sistemas democráticos, en la que las entidades del Estado ejecutan la gran mayoría de sus recursos en el último año de gobierno, dado que en el mismo se materializan sus procesos de planeación. Ante ello, en las entidades municipales, sobre todo en las que administran presupuestos reducidos, el proceso de planeación las obliga a ejecutar las principales iniciativas políticas en los últimos años de gobierno, dinamizando el gasto público y los ingresos tributarios. Así, puesto que el 2015 fue el último año del periodo administrativo de alcaldes, este condensa con mayor precisión la dinámica financiera de las entidades municipales.

\section{Cruce de variables según tamaño po- blacional. Desempeño fiscal versus desempeño integral}

De este modo, ya hechas las precisiones conceptuales, la Figura 2 representa los resultados conjuntos de desempeño fiscal e integral para los municipios de Boyacá en el año 2015, desagregados por el tamaño poblacional de los mismos. La distribución por colores permite diferenciar entre municipios de acuerdo con su demografía. Los puntos de municipios ubicados en la parte superior derecha de la gráfica son aquellos con óptimas condiciones fiscales y administrativas, contrario a aquellos que se encuentran en el sector inferior-izquierdo de la misma, con los rendimientos más bajos de la muestra. Los resultados se evalúan a continuación. 


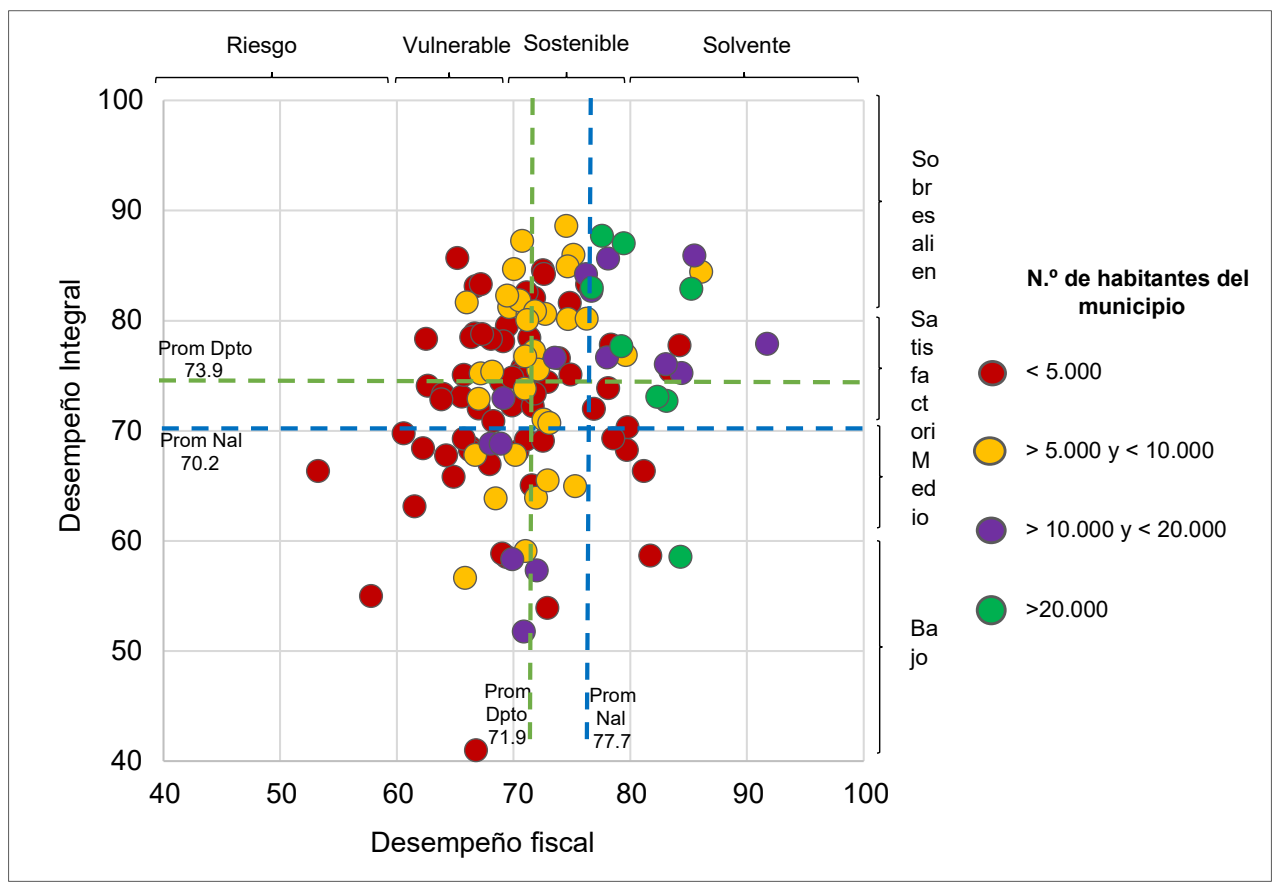

Figura 2. Desempeño integral vs desempeño fiscal municipal por tamaño poblacional. Boyacá 2015.

Fuente: elaboración propia, datos DANE (2005a), DNP (2016a) y DNP (2016b).

Resultados generales de desempeño fiscal en Boyacá

Como primer elemento de análisis, los resultados comparativos de Boyacá evidencian un bajo desempeño relativo en el indicador fiscal. Los municipios boyacenses manifestaron un indicador promedio en rango sostenible de 71.9, el cual es 5.8 puntos más bajo que el registrado en el promedio nacional de 77.7. Ante ello, 100 de las 123 entidades municipales boyacenses expresaron un desempeño fiscal por debajo de la media del país, tal como se muestra en la Figura 2 con la aglomeración de puntos hacia la izquierda de la línea azul vertical. En cuanto a las categorías de resultados establecidas por el DNP, 47 municipios se encuentran en condición vulnerable o de riesgo fiscal, por lo cual se caracterizan como de estructuras financieras con un alto nivel de dependencia fiscal o riesgo de déficit corriente ante las falencias en la generación de recursos propios (DNP, 2015a). En general, se manifiesta un rezago del departamento frente a la nación en términos financieros.

\section{Resultados de desempeño fiscal desa- gregados por tamaño poblacional}

Ahora, los resultados desagregados por tamaño poblacional reflejan que las 
fallas en el desenvolvimiento fiscal en Boyacá resultan más notorias en los municipios con estructuras demográficas reducidas. Así lo evidencia la Figura 2, cuyos puntos color rojo, correspondientes a municipios de menos de 5000 habitantes, se agrupan fundamentalmente hacia la izquierda de la gráfica, zona ya identificada como la de menor desempeño fiscal del estudio. De igual manera, en lo relacionado con las categorías de resultado del DNP, el $100 \%$ de los municipios del departamento clasificados como entidades en condición de riesgo y el $69 \%$ de aquellos que se ubican en el rango de vulnerables, pertenecen al grupo de entidades con menor tamaño poblacional en Boyacá (menos de 5000 personas).

También, el indicador promedio de desempeño fiscal por grupos de municipios nota un resultado diferencial, el cual desciende de forma progresiva entre más reducido es el tamaño poblacional. En efecto, para el caso de las entidades con más de 20000 habitantes (puntos color verde), observadas para el caso boyacense como ciudades, obtuvieron un indicador promedio de 80.99 , lo cual las identifica como alcaldías solventes. Para el segundo grupo de municipios, integrado por aquellos que tienen entre 10000 y 20000 pobladores (puntos color violeta), la cifra promedio fue de 76.20, calificados como sostenibles. En cuanto a los municipios con tamaños demográficos de entre 5000 y 10000 personas (puntos color amarillo), el resultado fue de 72,27 , identificados también como sostenibles, pero en valores más bajos que los del grupo anterior. Por último, para los municipios con menos de 5000 personas (puntos color rojo), el desempeño fiscal promedio fue de solo 69,64 , en condición promedio de vulnerabilidad, demostrando así una tendencia progresiva en el indicador ante el tamaño demográfico de los municipios. De esta manera es admisible atreverse a determinar que hay una relación de tendencia entre el desempeño fiscal de los municipios y su masa poblacional en Boyacá.

Más aún, los indicadores o variables desagregadas del desempeño fiscal profundizan la falencia relativa de los municipios con población reducida del departamento en materia financiera. En primer lugar, el promedio del indicador de autofinanciamiento de gastos de funcionamiento para los municipios de menos de 10000 habitantes es del $49 \%$, más alto que el $42 \%$ manifestado por el resto de entidades boyacenses; si se tiene en cuenta que los municipios de menor población son aquellos que ostentan una menor cantidad de ingresos corrientes de libre destinación (ICLD), este alto porcentaje en el indicador demuestra un reducido margen de flexibilidad para 
gastos administrativos o para inversión autónoma ${ }^{3}$. Por su parte, la proporción de las transferencias sobre los ingresos totales del mismo grupo de municipios es de $61 \%$, mientras que para las entidades con mayor masa poblacional es de $55 \%$, manifestando así la condición de dependencia de los primeros. De igual manera, la generación de recursos propios, que expresa la participación de los ingresos recaudados por la propia entidad sobre el total de ingresos corrientes, expresa un resultado de $42 \%$ para el primero, mientras que para el grupo de municipios con mayor tamaño poblacional es de $70 \%$ en promedio. Así, la inflexibilidad ante los ICLD, la alta dependencia fiscal y la reducida capacidad para recaudar tributos propios, son indicadores que demuestran también una heterogeneidad sustancial en el desempeño financiero de las entidades boyacenses de acuerdo con su estructura poblacional.

\section{Resultados generales de desempeño integral}

Por su parte, los resultados de desempeño integral parecen ser más homogéneos entre los distintos municipios del departamento según lo observado en la Figura 2, aunque revisando particularmente los componentes del mismo se demuestra que los indicadores de índole financiero conservan la divergencia en relación. Como muestra de ello, para el grupo de municipios con más de 20000 habitantes, el 73.9 de promedio general obtenido es influido positivamente por la variable de gestión administrativa y fiscal, la cual reporta para aquellos un valor promedio de 85.0. Por el contrario, las entidades de menor tamaño poblacional encuentran en los indicadores de eficiencia y de desempeño fiscal aquellas variables que limitan negativamente el indicador integral promedio con 54.1 y 69.6 respectivamente. El primero de estos indicadores acusa para tales municipios fallas en la relación de insumos y productos con las que se ejecutan las acciones gubernamentales, mientras que el segundo es un resumen del reservado ejercicio fiscal municipal en la vigencia, tanto en materia de ingresos, gastos, dependencia, autonomía, endeudamiento, entre otros elementos del ranking fiscal.

\section{Análisis de correlación lineal. Tama- ño poblacional-desempeño fiscal}

Ahora, como elemento final del análisis, la Figura 3 representa la correlación lineal entre las variables tamaño poblacional e indicador de desempeño fiscal para cada municipio del departamento, tomando la primera como independiente y la segunda como dependiente. Como se observa en la distribución, la relación lineal expresa una clara tendencia positiva entre las diferentes combinaciones

3 Ello se da porque la Ley 617 del 2000 dispone que las entidades deben solventar sus gastos de funcionamiento con sus ingresos corrientes que no tienen restricción de uso (ICLD). Ante ello, para los municipios de cuarta, quinta y sexta categoría, las alcaldías solo pueden usar hasta el $80 \%$ de sus ICLD en gastos de funcionamiento de la administración central. El total de ICLD de la entidad para una vigencia, restado los gastos de funcionamiento, corresponde a la suma de recursos de que dispone la misma para inversión autónoma. 
de los puntos. Es pertinente resaltar que por factibilidad metodológica y visual se han marginado del gráfico los cinco municipios del departamento cuya población es mayor a los 35000 habitantes, entendiendo que los mismos se comportan como datos demográficos atípicos en Boyacá, aunque ello no altera la tendencia observada. Así, se sustenta una relación de causalidad proporcional entre las variables, donde el tamaño demográfico de los municipios explica significativamente el desempeño financiero de las entidades territoriales.

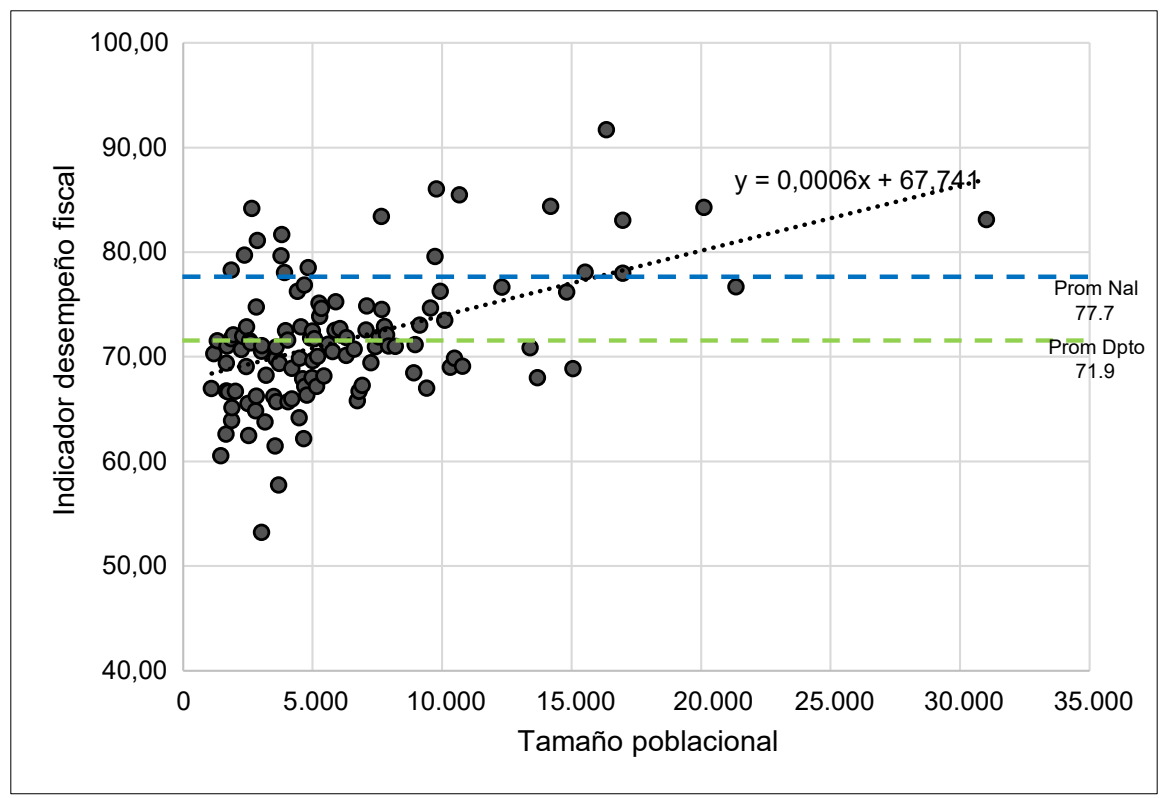

Figura 3. Correlación lineal - tamaño poblacional vs desempeño fiscal. Boyacá, 2015.

Nota: elaboración propia, datos DANE (2005a) y DNP (2016b).

Establecido lo anterior, se puede señalar que el desempeño financiero del departamento boyacense muestra una significativa tendencia de reducción en la medida que sus municipios son de menor tamaño poblacional, sustentando en cierta medida su rezago frente a los demás departamentos, ante un resultado promedio por debajo de la media nacional. Como se manifiesta en la Figura 2, los municipios pequeños expresados con puntos color rojo y amarillo, los cuales representan la gran mayoría en el departamento, presentaron indicadores relativamente bajos en desempeño financiero en promedio. De esta manera, la dependencia fiscal y la escasa generación de ingresos propios se decantan como problemáticas que se maximizan en entornos territoriales con bajo dinamismo demográfico, tal como se ha evidenciado en el caso de 
Boyacá. En consecuencia, si se toma en cuenta el hecho de que la gran mayoría de las transferencias de la nación son de naturaleza condicionada, es decir, que se transfieren con una destinación fija, la dependencia financiera y la escasez de ingresos propios consolidan una grave inflexibilidad en la ejecución del presupuesto de las entidades municipales, primordialmente en las de reducida masa demográfica. Sin ser el único factor desencadenante de los obstáculos en las finanzas públicas en Boyacá, el componente demográfico se suma como un ingrediente influyente de gran complejidad para el quehacer administrativo público en el territorio.

\section{RETOS DE LAS FINANZAS PÚ- BLICAS ANTE EL REDUCIDO TAMAÑO POBLACIONAL. SUB- VENCIONES POR RESULTADOS Y ENFOQUE TERRITORIAL}

Como medidas de reacción ante el escenario expuesto, la distribución equitativa de los recursos entre las regiones ha sobresalido como una disposición de vital relevancia para paliar las divergencias. Tal como lo expresa Restrepo (2015), basado en la teoría de las funciones fiscales de Musgrave, la distribución tiene como una de sus expresiones básicas la destinación equitativa de recursos públicos en el nivel territorial, como mecanismo para disminuir la pobreza en las regiones de menor progreso. Este argumento ha sido usado por las organizaciones de decisión fiscal en el Gobierno colombiano, hecho por el cual la distribución de recursos del Sistema General de Participaciones (SGP) en su componente de propósito general se realiza primordialmente por pobreza, tamaño poblacional y ruralidad (Ley 1176 de 2007). En consecuencia, Bonet y Ayala (2015) demuestran la preeminencia de un fenómeno progresivo de equidad fiscal entre los territorios del país, producido por la implementación de las transferencias del SGP. Tal condición es apoyada por Guerrero (2014), quien sustenta a través de análisis de coheficientes como el de Gini, Theil o Sigma, que la descentralización fiscal ha decantado en Boyacá una tendencia progresiva hacia la convergencia intermunicipal. Los argumentos fundamentales de dichos autores se desenvuelven a partir de la horizontalidad con la cual se han ejecutado las políticas de transferencias intergubernamentales desde el proceso de descentralización, que han conducido a equiparar los ingresos de las diferentes entidades locales.

No obstante, si se extiende el análisis hasta evaluar el desempeño de tales administraciones, se devela una gran divergencia en los indicadores resultantes de las distintas entidades, a pesar de su relativo aumento en los ingresos totales, minando en cierta medida la tesis presentada de equidad fiscal. En efecto, el modelo de transferencias usado en la descentralización colombiana ha dotado de recursos a los municipios para que ejerzan su actividad pública, pero ello no garantiza ni conduce a que las mismas se desenvuelvan con eficiencia 
y solvencia, entendiendo que el modelo privilegia fundamentalmente la pobreza y no el desempeño como criterios de distribución de las subvenciones (Velasco, 2007). Además, la condicionalidad con la que se transfieren los recursos nacionales priva a los municipios de la dirección sobre la destinación de los mismos, alterando el principio de autonomía fiscal. Según Wiesner (2002), el éxito de una real descentralización no se efectúa en la medida que las transferencias intergubernamentales aumenten, sino que se materializa cuando los ingresos propios de las administraciones locales se incrementan en una mayor proporción a los recursos externos. De manera general, el modelo implementado en Colombia reduce la inequidad en términos de riqueza bruta, pero no garantiza la maximización de las aptitudes financieras de las entidades locales, tal como ha sucedido en el escenario territorial.

Surge así la necesidad de reorientar los criterios de distribución de las subvenciones, de manera que promuevan el desempeño y los resultados financieros más allá de las condiciones de vulnerabilidad o pobreza. Se manifiestan alternativas de evaluación del desempeño fiscal local y de nuevos criterios para la destinación de transferencias intergubernamentales, los cuales tienden a privilegiar la planeación y la generación de resulados medibles. Una de ellas es la presentada por el Programa del Gasto Público y Rendición de Cuentas (PEFA), el cual expresa que la evaluación fiscal de las entidades debe tener en cuenta, más allá de su nivel de ingresos o de dependencia, elementos como la confiabilidad del presupuesto, la transparencia de las finanzas públicas, la gestión de activos y pasivos, la estrategia y presupuestación basada en políticas, entre otros elementos que privilegian la gestión eficiente y planeada del presupuesto (PEFA, 2016).

Por su parte, Boadway y Shah (2009) afirman que las tranferencias intergubernamentales deben tener una condicionalidad por resultados, es decir, que el otorgamiento de subvenciones ficales debe ir acompañado de un proceso de evaluación sobre los efectos del uso de los recursos transferidos a las localidades, de tal manera que los mismos sean un relevante criterio de distribución. Un país que ha demostrado relativos avances en este aspecto es Ecuador, el cual ha adherido entre los criterios de distribución de transferencias la variable denominada "Logros en el mejoramiento de los niveles de vida", la cual se mide a través de la dismunición interanual del NBI; a pesar de que su peso relativo sobre la distribución aún es bajo (5\%), el régimen ecuatoriano es un gran ejemplo de la distribución de recursos por resultados o impacto (SENPLADES, 2012). Así, implementando mecanismos de evaluación fiscal y distribución de recursos nacionales que privilegien la generación de resultados, las entidades municipales tenderían, en el mejor de los casos, a conformar estrucuturas de 
recaudo y gasto social óptimo ante un régimen de distribución meritorio.

Por último, es necesario aclarar que la implementación del modelo de subvenciones por desempeño y resultados es insuficiente ante fenómenos problemáticos como el despoblamiento presentado en el caso boyacense. Una de las características sustanciales del mismo en un territorio es que se desenvuelve por una serie compleja de fenómenos sociales, que en la mayoría de los casos superan las facultades gubernativas de las entidades estatales, sobre todo en ambientes locales. Ante ello, el fortalecimiento de las atribuciones fiscales locales por medio del modelo propuesto debe ir acompañado de una orientación política macro en la que se aborden las dintintas problemáticas sociales que conducen a la emigración de la población en el territorio, particularmente en los municipios con mayor ruralidad. Para ello, las administraciones deben dimensionar la complejidad cultural, económica, social y geográfica de las localidades marginadas, para emprender medidas políticas mancomunadas que estimulen los sectores económicos del municipio, especialmente la maximización de las atribuciones productivas rurales de las regiones. Estas orientaciones a largo plazo son insumos de vital importancia para estimular el sistema tributario municipal y fortalecer las finanzas públicas de organizaciones estatales históricamente marginadas del crecimiento económico.

\section{CONCLUSIONES}

El documento ha puesto en evidencia una relación tendencial entre el tamaño poblacional de los municipios y el desempeño financiero de las entidades públicas, ejemplificada en el caso particular del departamento de Boyacá. Las grandes falencias de autonomía fiscal y desenvolvimiento tributario propio, características del proceso de descentralización fiscal colombiano, encuentran puntos críticos en territorios con reducida dinámica demográfica. El desenvolvimiento hacendístico de estas entidades locales se ha visto rezagado ante un sistema de descentralización que favorece el principio de equidad territorial y la implementación del modelo del subsidio a la demanda, pero que no establece criterios de distribución que premien a las entidades por su desempeño financiero.

Así, en un primer momento, las cifras censales evidencian que el departamento boyacense ha padecido un proceso histórico de despoblación en provincias periféricas, ante lo cual se ha gestado una estructura demográfica de pequeños municipios. Entidades específicas han reducido su tamaño poblacional desde mediados del siglo XX en porcentajes cercanos al $50 \%$, mientras que en regiones centrales se ha incrementado al doble. Ante esta condición, se consagra un entorno diferencial entre las distintas provincias y municipalidades del departamento, el cual produce una 
condición inicial heterogénea en el desenvolvimiento de las administraciones.

Dadas estas condiciones, el análisis de correlación lineal ha demostrado que la estructura fiscal municipal del departamento boyacense está fuertemente influenciada por este escenario demográfico, de tal forma que los indicadores de desempeño financiero del departamento tienden a rezagarse ante las reducidas estructuras tributarias de sus municipios con menor masa poblacional. En este sentido, el análisis sustenta que la gran mayoría de municipios boyacenses con menos de 10000 habitantes manifiestan problemas de autonomía financiera y de excesiva dependencia de los recursos exógenos. Así, las problemáticas más dicientes del proceso de descentralización fiscal se ven fuertemente afectadas por sistemas de ciudades pequeñas $\mathrm{y}$ regiones que padecen trampa de pobreza. Se conforma así un cúmulo de circunstancias sociodemográficas que alteran el devenir financiero de las entidades, rezagan su desarrollo y debilitan la democracia descentralizada.

Ante ello, se plantea la necesidad de establecer criterios meritorios de distribución de las transferencias inter- gubernamentales, los cuales estimulen de manera más pronunciada el desenvolvimiento de las facultades fiscales de las entidades locales. Sin perder de vista el carácter equitativo de tales criterios, las entidades de orden nacional deben profundizar en los mecanismos de evaluación de los procesos de planeación financiera y del resultado de impacto generado con el uso de los recursos exógenos, considerando los mismos para definir un significativo porcentaje de las subvenciones por criterio general. También, ante fenómenos como la despoblación, el fortalecimiento de las finanzas municipales requiere de una serie de medidas políticas a largo plazo que incentiven el desprendimiento de las facultades territoriales de las regiones.

\section{AGRADECIMIENTOS}

El autor agradece a la Escuela Superior de Administración Pública, en especial a los docentes Orlando Velasco Ulloa y Julio Cesar Caro Moreno, por sus orientaciones temáticas y metodológicas sobre el documento. También se agradece a los pares evaluadores por sus valiosas sugerencias y aportes. Así mismo, asume la responsabilidad sobre las opiniones contenidas en el artículo. 


\section{REFERENCIAS}

Asela , J. E. (2015). Realidad fiscal de los municipios de Santander, en el marco del proceso de descentralización fiscal en Colombia, 2004-2012. Apuntes del CENES, 34(60), 181-214. https://doi.org/10.19053/22565779.3779

Boadway, R. \& Shah, A. (2009). Transferencias fiscales intergubernamentales. Principios y práctica. Colombia: Banco Mundial. https://doi. org/10.1596/978-9-5883-0753-4

Bonet, J. \& Ayala, J. (2015). Transferencias intergubernamentales y disparidades fiscales horizontales en Colombia. Economía Regional, Banco de la República, (231). https://doi.org/10.32468/dtseru.231

CEPAL. (2016). Panorama fiscal de América Latina y el Caribe 2016. Las finanzas públicas ante el desafío de conciliar austeridad con crecimiento e igualdad. Santiago de Chile: División de Desarrollo Económico de la CEPAL.

Congreso de la República de Colombia. (2007, 27 de dic.). Ley 1176. Diario Oficial, (46.854). Recuperado de http://www.secretariasenado.gov.co/senado/basedoc/ ley_1176_2007.html

Congreso de la República de Colombia. (2012, 6 de jun.). Ley 1551. Diario Oficial, (48.483). Recuperado de http://www.secretariasenado.gov.co/senado/basedoc/ ley_1551_2012.html

De Lucio, J. J. (11 de 1997). Geografía económica: aglomeración, localización y externalidades. Recuperado de http://documentos.fedea.net/pubs/dt/1997/dt1997-21.pdf

Delgado, S. T. \& Acero, L. E. (2015). Análisis de dependencia fiscal de los municipios de sexta categoría del departamento de Boyacá, durante el periodo 1996-2012. Apuntes del CENES, 34(60), 215-246. https://doi.org/10.19053/22565779.3780

Departamento Administrativo Nacional de Estadística -DANE-. (2005a). Estimaciones de población 1985-2005 y proyecciones de población 2005-2020 total municipal por área. Recuperado de https://www.dane.gov.co/index.php/ estadisticas-por-tema/demografia-y-poblacion/proyecciones-de-poblacion 
Departamento Administrativo Nacional de Estadística -DANE-. (2005b). Cuadro censo 2005. Recuperado de https://www.dane.gov.co/index.php/ estadisticas-por-tema/demografia-y-poblacion/censo-general-2005-1

Departamento Nacional de Planeación -DNP-. (2015a). Resultados desempeño fiscal de los departamentos y municipios 2014. Recuperado de https://www.dnp.gov. co/programas/desarrollo-territorial/Estudios-Territoriales/Indicadores-y-Mediciones/Paginas/desempeno-fiscal.aspx

Departamento Nacional de Planeación -DNP-. (2015b). Resultados de desempeño integral de los departamentos y municipios de la vigencia 2014. Recuperado de https://www.dnp.gov.co/programas/desarrollo-territorial/Estudios-Territoriales/Indicadores-y-Mediciones/Paginas/desempeno-integral.aspx

Departamento Nacional de Planeación -DNP-. (2016a). Resultados de desempeño integral de los departamentos y municipios de la vigencia 2015 (Anexo datos). Recuperado de https://www.dnp.gov.co/programas/desarrollo-territorial/Estudios-Territoriales/Indicadores-y-Mediciones/Paginas/desempeno-integral.aspx

Departamento Nacional de Planeación -DNP-. (2016b). Resultados de desempeño fiscal de los departamentos y municipios en la vigencia 2015. Anexo. Recuperado de https://www.dnp.gov.co/programas/desarrollo-territorial/Estudios-Territoriales/Indicadores-y-Mediciones/Paginas/desempeno-fiscal.aspx

Galvis, L. A. \& Meisel, A. (2011). Persistencia de las desigualdades regionales en Colombia: un análisis espacial. En L. Bonilla, L. A. Galvis, J. Romero \& A. Meisel, Dimensión regional de las desigualdades en Colombia (pp. 1-33). Bogotá: Banco de la República. https://doi.org/10.32468/dtseru.120

Giraldo, C. (2009). Finanzas públicas en América Latina: la economía política. Bogotá: Ediciones Desde Abajo.

Guerrero, P. A. (2014). Finanzas públicas y desigualdad fiscal en los municipios de Boyacá, 1985-2010. Apuntes del CENES, 33(57), 177-222. https://doi. org/10.19053/22565779.2908

Muñoz, C. (2013). Boletín de finanzas públicas territoriales, gobiernos centrales departamentales, y gobiernos centrales municipales incluyendo capitales, cierre a 
diciembre de 2012. Perfil de Coyuntura Económica. Universidad de Antioquia, (22), 141-177.

PEFA. (2016). Marco de referencia para la evaluación de la gestión de las finanzas públicas. Washington, DC: Secretariado PEFA.

Pening, J. P. (2003). Evaluación del proceso de descentralización en Colombia. Economía y Desarrollo, 2(1), 123-149.

Pineda, J. (2016). Despoblamiento en Boyacá: retos y desafios. Bogotá: Escuela Superior de Administración Pública -ESAP-.

Pinilla, D., Jiménez, J. D. \& Montero, R. (2015). La descentralización fiscal en América Latina. Balance de un proceso. Revista de Economía Institucional, 17(33), 133-166. https://doi.org/10.18601/01245996.v17n33.06

Restrepo, J. C. (2015). Hacienda Pública (10 ed.). Bogotá: Universidad Externado de Colombia.

Sánchez, C. A., Naranjo, R. \& Rincón, E. (2005). Ajuste estructural de las finanzas públicas 1998-2004. Medellín: Biblioteca Jurídica Diké, Universidad del Rosario.

SENPLADES. (2012). Guía metodológica para el cálculo de la distribución de los recursos fiscales para los gobiernos autónomos descentralizados. Quito: Técnica.

Velasco, O. (2007). Finanzas públicas municipales. Bogotá: Universidad Externado de Colombia.

Wiesner, E. (2002). Transferencias, incentivos y endogeneidad del gasto territorial. Archivos de Economía, (174). 



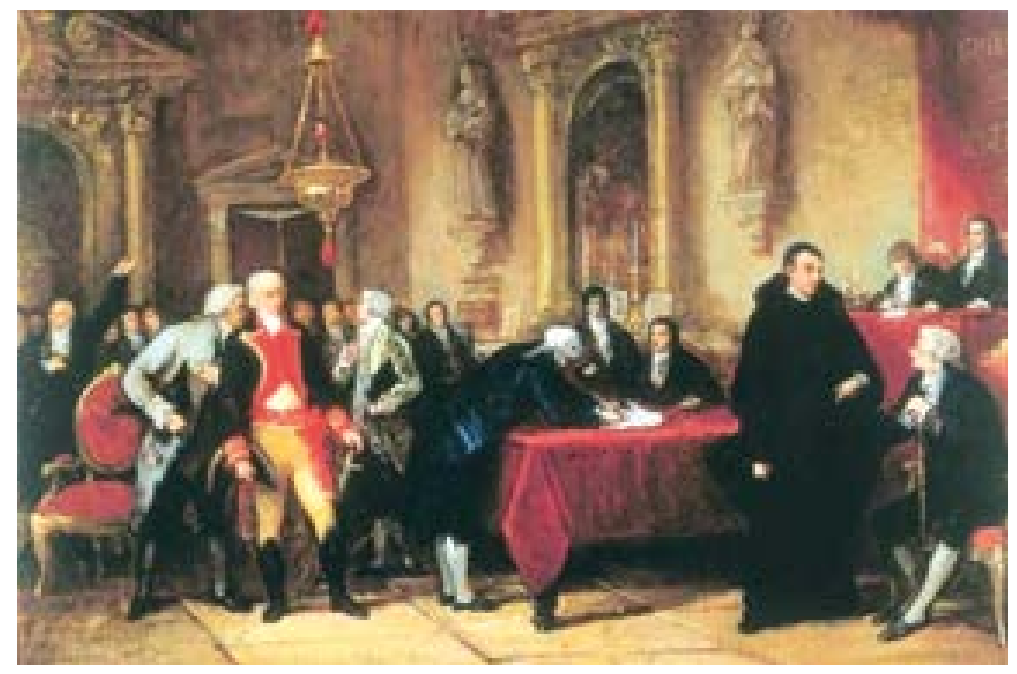

Boceto para la Fima del Acta de Independencia

\section{DIRECTRICES PARA AUTORES}


